\title{
Oral ulcerations as the first manifestations of acute leukemia: A case report
}

\author{
Somayeh Alirezaei ${ }^{1}$, Mahin Bakhshi ${ }^{2}$, Jamileh-Bigom Taheri ${ }^{2}$, Ahmad R. Mafi ${ }^{3 *}$, Omid Moghaddas $^{4}$ \\ ${ }^{1}$ Department of Oral and Maxillofacial Medicine, Dental School, Islamic Azad University, Tehran, Iran \\ ${ }^{2}$ Department of Oral and Maxillofacial Medicine, Dental School, Shahid Beheshti University of Medical Sciences, Tehran, Iran \\ ${ }^{3}$ Jorjani Cancer Center, Imam Hossein Hospital, Shahid Beheshti University of Medical Sciences, Tehran, Iran \\ ${ }^{4}$ Department of Periodontology, Dental School, Islamic Azad University, Tehran, Iran \\ Email: ahmadrmafi@yahoo.com
}

Received 20 November 2013; revised 21 December 2013; accepted 31 December 2013

Copyright (C) 2013 Somayeh Alirezaei et al. This is an open access article distributed under the Creative Commons Attribution License, which permits unrestricted use, distribution, and reproduction in any medium, provided the original work is properly cited.

\begin{abstract}
Acute myeloblastic leukemia (AML) is a highly fatal malignant bone marrow disease. Physicians, dentists and all other healthcare professionals should be aware of sinister oral signs and symptoms in order to early diagnosis and referral of patients. Here we report a case of AML who presented with oral ulcers. Ulcers developed after a parrot bite, which initially misled the physicians. Unfortunately our patient did not survive, but early diagnosis and prompt investigation and treatment can be life-saving in many other similar cases.
\end{abstract}

Keywords: Acute Leukemia; Oral Ulcers

\section{INTRODUCTION}

A 48-year-old female presented to the General Dental Clinic of our hospital with chief complaint of painful ulcers in gingiva and tongue that had developed three weeks before. The lesion had started as a small ulcer in her tongue, but gradually enlarged and after a couple of days gingival ulcers developed.

On history taking, she gave a history of lethargy, mild fever and slight weight loss. She also mentioned that she kept a parrot in her house and she often fed the bird mouth to mouth, and remarked that the lesion first started when the parrot accidentally bit her tongue a couple of weeks before. Furthermore, she remarked that her sister also fed the parrot mouth to mouth, and she had developed similar ulcers that had healed spontaneously. The rest of past medical history was unremarkable.

Based on this history, with the primary diagnosis of a zoonotic disease, the patient was referred to oral medi-

"Corresponding author. cine department for consultation with an oral medicine specialist.

On arrival to our department, it could be seen that she was pale and ill. Extra-oral examination revealed swelling and tenderness to palpitation of the face.

Intra-orally, bilateral palatal and lingual gingiva appeared to be mildly swollen, glazed, devoid of stippling and spongy in consistency. The color of the marginal and papillary gingiva was dark red. Several ulcers were observed on gingival and buccal mucosa. Ulcers were covered by a necrotic slough and surrounded by an erythematous margin. A necrotic ulcer was also seen on left lateral border of tongue (Figures $\mathbf{1}$ and 2).

As part of departmental routine, first we started investigating the disease with a systemic approach, and lab tests were ordered as a part of this approach.

Haematology results revealed anemia $(\mathrm{Hgb}=10.2$ $\mathrm{g} / \mathrm{dL})$, thrombocytopenia $\left(\mathrm{Plt}=49,000 / \mathrm{mm}^{3}\right)$, and marked leukocytosis $\left(\mathrm{WBC}=133,000 / \mathrm{mm}^{3}\right)$. ESR was also grossly elevated. Examination of the blood smear revealed large cells with large nucleus with distinct nucleoli. Bone marrow biopsy revealed the diagnosis of acute myelomonocytic leukemia (AML-M4).

The patient was referred to the hematology department. Chemotherapy started, which was not successful. She went to coma after the second cycle of chemotherapy and passed away a couple of days later.

\section{DISCUSSION}

There are several etiologies for oral lesions and ulcers. The majority of these lesions and ulcers are benign and often self-limiting; therefore, the art of a physician is to diagnose those sinister lesions that can be life-threatening. Lesions of different etiologies have different characteristics, and proper knowledge of these characteristics 


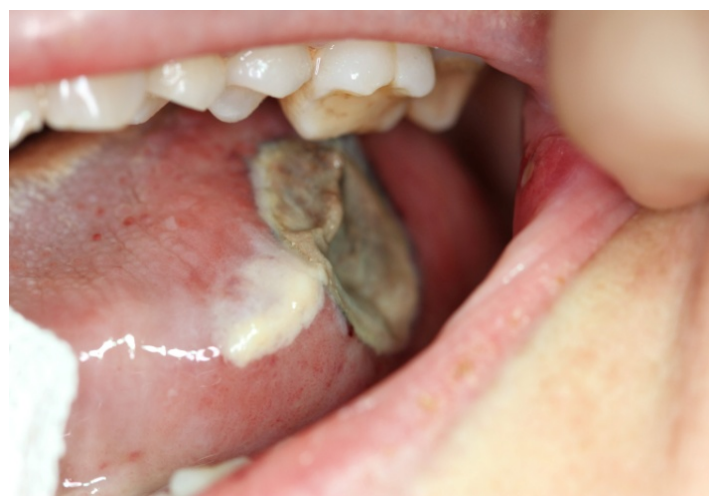

Figure 1. Lesion in left lateral border of tongue.

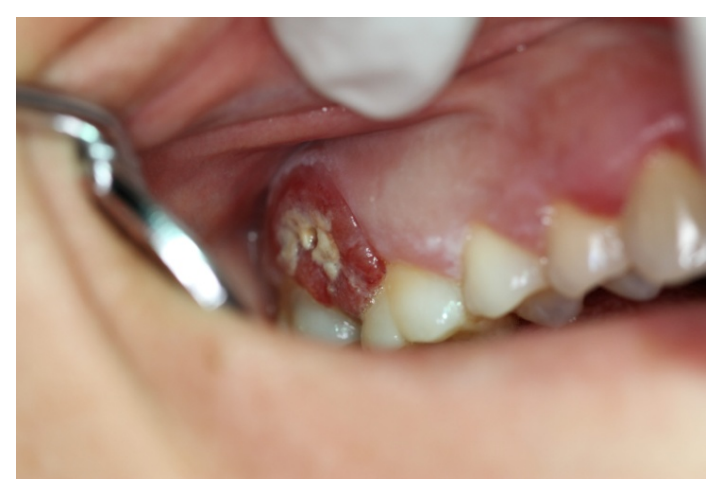

Figure 2. Lesion in right upper gingival.

is essential for health care professionals who are involved in treating oral lesions. For example, while genetically induced gingival overgrowth is normal colored and firm, gingival overgrowth due to blood dyscrasias are edematous, soft, tender to touch and show tendency to bleed $[1,2]$.

Oral lesions are relatively common in leukemias, as a part of a widespread disease. However, oral ulcers and lesions could be the first presentation of the disease [2,3].

Leukemia is a broad term covering a spectrum of diseases. Clinically and pathologically, leukemia is subdivided into chronic and acute forms. Chronic leukemias involve relatively well differentiated leukocytes, are slow in onset and typically take months or years to progress. Hence, immediate treatment sometimes is not necessary, and patients can be monitored for some time before treatment to ensure maximum effectiveness of therapy $[2,4]$.

On the other hand, acute leukemias are characterized by a rapid and uncontrolled proliferation of poorly differentiated blast cells, for which immediate treatment is required. They are abrupt in onset, and are aggressive and rapidly fatal if left untreated. Oral manifestations are more common in acute leukemias [5].

One of the sinister and fatal etiologies of oral ulcers and lesions, is Acute Myeloid leukemia (AML), mainly acute monocytic (M5) acute myelomonocytic (M4), and acute myelocytic (M1, M2) leukemias. Oral lesions may be the presenting feature of acute leukemias and are therefore important diagnostic indicators of the disease $[4,6]$.

Most signs and symptoms of AML are caused by the replacement of normal blood cells with leukemic cells. They usually present with signs and symptoms of bone marrow failure, including anemia, infection, and bleeding. At first, symptoms are non-specific; such as bone pain, joint pain, or other flu-like symptoms, and patients usually seek medical help because of these constitutional symptoms that have lasted more than usual. Oral cavity usually is involved as part of a widespread disease; however, oral ulcers can be the first presentation of the disease which can lead physicians to make exact diagnosis [5].

Most of the time, the patients with an oral lesion first consult their dentist, who-with proper knowledge and awareness of potentially fatal etiologies - can play a vital role in early diagnosis of the disease.

According to various reports, the most common presentation of AML in the oral cavity include gingival enlargement, local abnormal color orgingival hemorrhage, petechiae, ecchymoses, mucosal ulceration and oral infections $[3,6]$.

The fact that oral lesions are sometimes the first manifestation of life-threatening diseases implies that dental professionals must be familiar with the clinical manifestations of systemic diseases [1,5].

In our case, the history of feeding the bird and presence of similar oral ulcers in patient's sister was quite misleading and drew all attentions to a zootonic disease as a possible cause of the disease. Unfortunately, our patient did not survive, as the overall prognosis of AML is poor, however, in case of a potentially curable disease, early diagnosis of an oral lesion can be life-saving.

\section{CONCLUSION}

This case (and other similar cases) underlies the importance of oral signs and symptoms as indicators of a systemic disease. Apart from physicians who generally diagnose acute leukemias based on systemic manifestations, dentists also can play an important role in diagnosing the disease, especially in patients who present with an oral lesion as the first manifestation of the disease. Although our patient had a poor prognosis anyway, early diagnosis and referral could be life-saving for many other similar cases.

\section{REFERENCES}

[1] Demirer, S., Özdemir, H., Şencan, M. and Marakoglu, I. (2007) Gingival hyperplasia as an early diagnostic oral manifestation in acute monocytic leukemia: A case report. 
European Journal of Dentistry, 12, 111-114.

[2] Parisi, E., Draznin, J., Stoopler, E., Schuster, S.J., Porter, D. and Sollecito, T.P. (2002) Acute myelogenous leukemia: advances and limitations of treatment. Oral Surgery, Oral Medicine, Oral Pathology, Oral Radiology and Endodontology, 93, 257-263.

http://dx.doi.org/10.1067/moe.2002.121988

[3] Kinane, D.F. and Marshall, G.J. (2001) Periodontal manifestations of systemic disease. Australian Dental Journal, 46, 2-12.

http://dx.doi.org/10.1111/j.1834-7819.2001.tb00267.x

[4] Wu, J., Fantasia, J.E. and Kaplan, R. (2002) Oral manifestations of acute myelomonocytic leukemia: A case re- port and review of the classification of leukemias. Journal of Periodontology, 73, 664-668.

http://dx.doi.org/10.1902/jop.2002.73.6.664

[5] Chavan, M., Subramaniam, A., Jhaveri, H., Khedkar, S., Durkar, S. and Agrwal, A. (2010) Acute myeloid leukemia: A case report with palatal and lingual gingival alterations. Brazilian Journal Of Oral Sciences, 9, 67-69.

[6] Dean, A.K., Ferguson, J.W. and Marvan, E.S. (2003) Acute leukaemia presenting as oral ulceration to a dental emergency service. Australian Dental Journal, 48, 195197.

http://dx.doi.org/10.1111/j.1834-7819.2003.tb00032.x 